\title{
Advanced Manifolds for Improved Solid Oxide Electrolyzer Performance
}

\author{
Diane L. Linne, ${ }^{1}$ Maria A. Kuczmarski, ${ }^{2}$ James Christopher Johnston, ${ }^{3}$ Serene C. Farmer, ${ }^{4}$ and Robert D. \\ Green $^{5}$ \\ NASA Glenn Research Center, Cleveland, OH, 44135, USA
}

Subodh K. Mital, ${ }^{6}$ and John A. Setlock ${ }^{7}$

University of Toledo, Toledo, $\mathrm{OH}, \mathrm{USA}$

\begin{abstract}
An investigation was conducted to see if an additive manufacturing process could be used to fabricate more efficient manifold designs for improved flow, reduced stresses, and decreased number of joints to be sealed for a solid oxide electrolyzer used to convert carbon dioxide to oxygen. Computational flow and mechanical modeling were conducted on a NASA Glenn Research Center patented cell and stack design with the potential to achieve a mass reduction of up to 75 percent. Various manifold designs were modeled, and two were downselected to be fabricated and tested. A baffled manifold design directed incoming flow more effectively into the flow channels compared to the original design, where the flow spent more time within the manifold itself. Flow measurements indicated some non-uniformity of flow across the channels at higher flow rates, which were not predicted by the model. Some possible explanations for the differences are discussed.
\end{abstract}

\section{Nomenclature}

$\begin{array}{ll}\text { AM } & =\text { additive manufacturing } \\ \text { BSC } & =\text { bi-electrode supported cell } \\ \text { ISRU } & =\text { in-situ resource utilization } \\ \text { SOE } & =\text { solid oxide electrolyzer / electrolysis } \\ \text { YSZ } & =\text { yttria stabilized zirconia }\end{array}$

\section{Introduction}

Due to the cost and weight considerations of future deep space explorations, harnessing of in-situ resources to generate required products, called In-Situ Resource Utilization (ISRU), is becoming increasingly important. Production of oxygen from atmospheric carbon dioxide for ascent propulsion and life support has been identified as an enabling technology for crewed Mars missions [1,2]. Solid oxide electrolyzers (SOE) are the leading technology candidate for producing oxygen from the carbon dioxide in the Mars atmosphere. To produce sufficient oxygen propellant for a Mars ascent vehicle, the SOE stacks must maintain production performance for 500 days or more while operating at greater than $800{ }^{\circ} \mathrm{C}$. If the carbon dioxide fed to the system does not flow uniformly throughout the stack, the full oxygen production capability of the stack will not be achieved. If any cracking in the stack components occurs due to launch or landing mechanical loads or during repeated thermal cycles, gases will leak which could severely impact the system operation and efficiency.

While most solid oxide stacks are formed by interspersing metallic manifolds between the ceramic electrolyte layers [3], the NASA Glenn Research Center has developed a patented cell and stack design with the potential to

\footnotetext{
${ }^{1}$ Senior Research Engineer, Propulsion Division, AIAA Associate Member.

${ }^{2}$ Research Materials Engineer, Materials and Structures Division, AIAA Senior Member.

${ }^{3}$ Chemical Engineer, Materials and Structures Division, AIAA Senior Member.

${ }^{4}$ Materials Research Engineer, Materials and Structures Division.

${ }^{5}$ Aerospace Engineer, Propulsion Division.

${ }^{6}$ Senior Research Associate, Mechanical, Industrial, and Manufacturing Engineering Dept.

${ }^{7}$ Senior Research Associate, Mechanical, Industrial, and Manufacturing Engineering Dept.
} 
reduce mass by as much as 75 percent. This is accomplished by eliminating the metal manifolds and frames, which constitute up to 70 percent of the stack mass, and replacing them with a thin zirconium oxide ceramic membrane with the gas channels moved into the electrodes. The cell design, called a bi-electrode supported cell (BSC) because it is structurally symmetrical and has identical gas channels on the cathode and anode sides, is a cross-flow design where

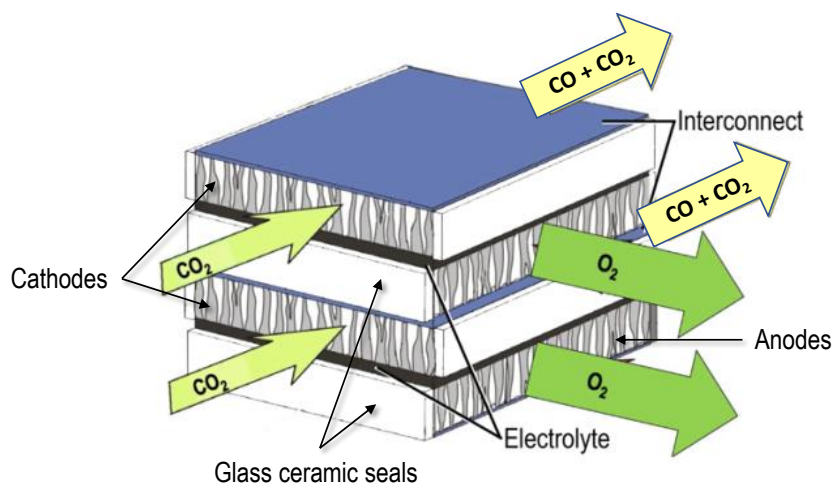

Fig. 1 BSC stack diagram of two cells stacked in series depicting the gas flow paths and sealing configuration in a cross-flow stack design.

the cathode and anode gas pathways are $90^{\circ}$ to each other [4]. Figure 1 displays a diagram of two cells with the seal configuration to achieve this cross-flow design. Ceramic inlet and outlet manifolds fabricated using a slip-cast method are sealed to the outside of the stack. Conventional state-of-the-art fuel cell or electrolyzer stacks with multiple cells require a sealing material that is applied at each interconnect layer and a significant compressive clamping force to form seal integrity. Because ceramic layers have some undulations, sealing the stack is a major concern. Clamping forces can help in that regard but high clamping force can also cause cracking of the electrolyte layer itself forming a new leak path. NASA's BSC design does not require a clamping force thus simplifying the design and construction of the stack. While successful hermetic seals have been achieved around the BSC manifolds, slip-casting allows for only very simplistic manifold designs and requires that the gas inlet and outlet tubes be a separate part, resulting in additional sealing joints and challenges. Therefore, the use of additive manufacturing (AM) was investigated to determine its potential to enable more efficient manifold designs for improved flow and reduced stresses, while reducing the number of joints to be sealed.

\section{Fluid Modeling and Design}

The BSC solid oxide electrolyzer 3-cell stack utilized in this study is shown in Fig. 2. Each cell consists of an anode / electrolyte / cathode layer, and the three cells were stacked with a metallic interconnect layer between each. To enable gas flow to the electrolyte, the anode and cathode layers are formed with high porosity, approximately 35 percent, and have laser-etched channels to further facilitate gas flow (Fig. 2). Manifolds direct the flow of gas into and out of the channels in each cell of the electrolyzer cell stack. The 3-cell stacks are approximately $5 \mathrm{~cm}$ on each side and $0.5 \mathrm{~cm}$ tall.

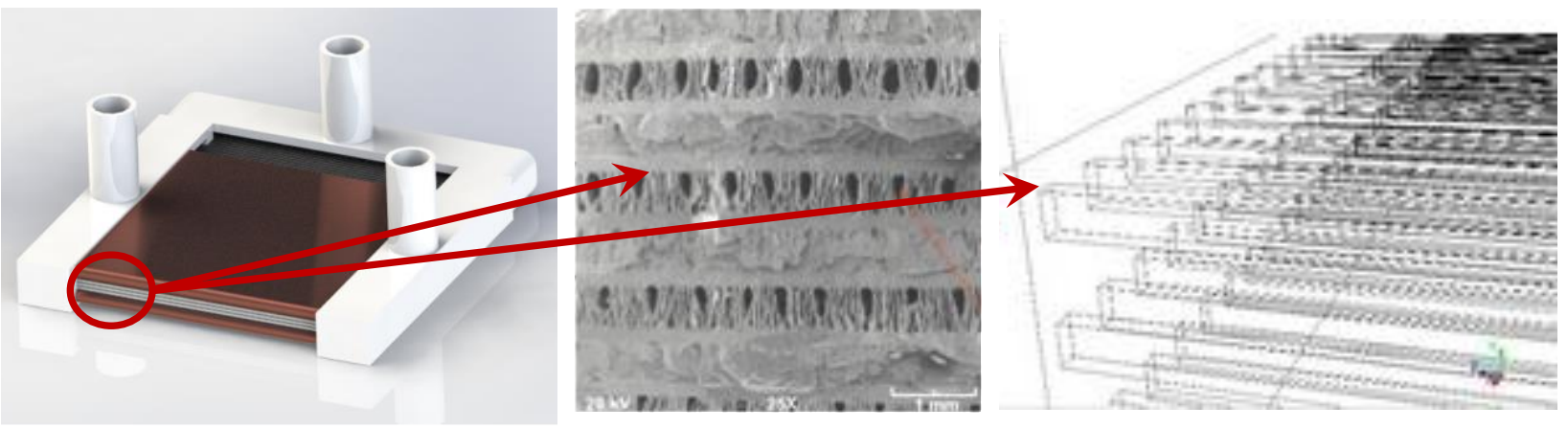

Fig. 2 BSC solid oxide 3-cell stack (left), magnified view of porous and laser-etched flow channels (middle), and CFD-rendered flow paths (right).

The computational fluid dynamics (CFD) code ANSYS® FLUENT, release 16.1 was used to model the fluid flow through the stack with the goals of: 1) visualizing the predicted flow through the stack; and 2) optimizing the manifold design to ensure uniform flow throughout the stack. Visualization within a system often leads to a better understanding of what is occurring and how to improve system performance. In systems such as the solid oxide electrolyzer stacks, visualization of internal flows is experimentally impractical. Computer modeling provides a far more efficient method of examining the flow of gas through the system. 
Seven different manifolds were designed for the three-cell stack (Fig. 3): 1) a large diameter (6.35 mm) tube centered on the top face of the original manifold; 2 ) a large diameter tube at the extreme edge of the top face of the original manifold; 3) a small diameter $(3.175 \mathrm{~mm})$ tube centered on the front face of the original manifold; 4) a small diameter tube centered on the front face of the original manifold with a diverter pin inside the body of the manifold, centered on the tube; 5) a small diameter tube centered on the side face of the original manifold; 6) two small diameter tubes, one on each side face of the original manifold; and 7) a large diameter tube centered on a wedge manifold with internal baffles. Design 6 was not modeled because it doubled the number of manifold connections that would have to be made which would make constructing the stack more difficult and increase the chances of leakage and/or failure at a joint.

The three-cell stack was modeled with 94 channels on the carbon dioxide side (cathode) of each of the three electrolyte layers. Cases were run at room temperature for comparison to flow tests described later, and at operating temperature $\left(850{ }^{\circ} \mathrm{C}\right)$ to
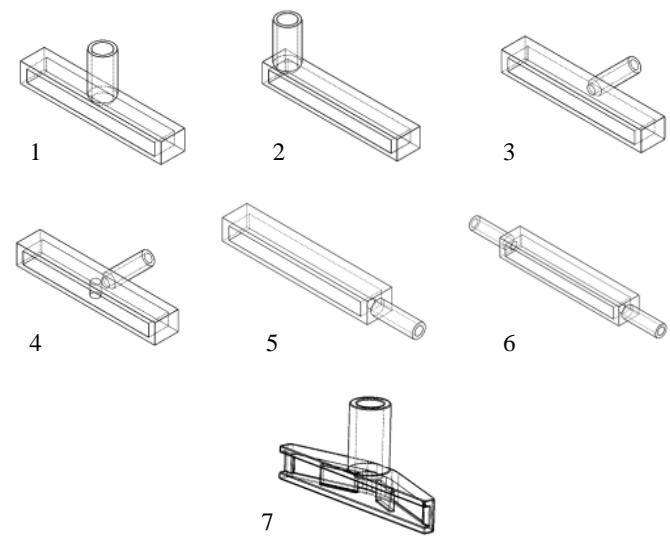

Fig. 3 Original and new manifold designs evaluated (see text for description). identify any changes that may occur at the higher temperatures caused by changing gas properties. The results of the computational modeling demonstrated that flows through the stack were insensitive to the manifold design, operating temperature, channel diameter, and number of cells in the stack. This is likely because of the extremely small area of the channels compared to the manifold. The open area of the channels was 5 to 9 percent of that of the manifold. There was, in every case, sufficient resistance to the flow of gas into the channels to mask any difference in the uniformity of flow in the manifolds. To decrease the resistance in the cathode channels, the original manifold design was also run with larger diameter channels, with similar results. It was noticed that the original manifold had about a three-times longer predicted residence time than the improved design of manifold 7. This indicates that if future developments decrease
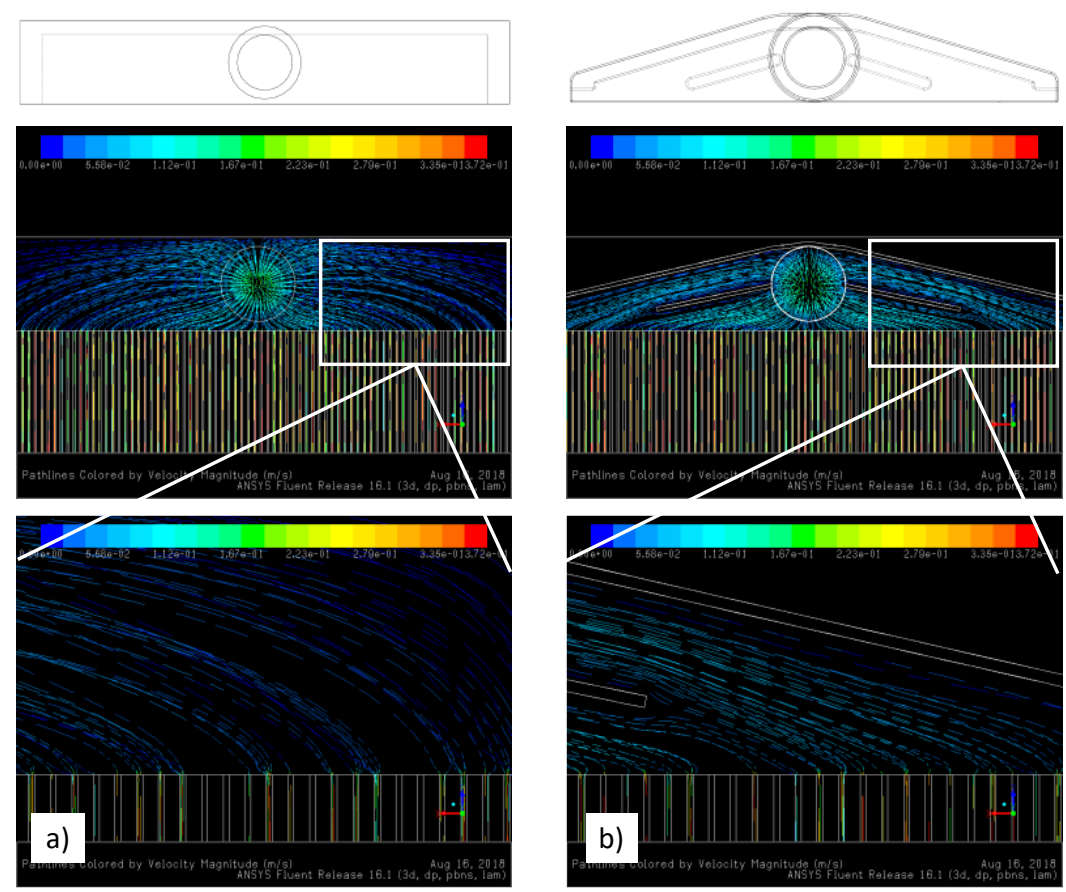

Fig. 4 Flow patterns in manifolds showing relative velocity for a) original manifold design; b) baffled manifold design the flow resistance in the stack, the improved design is also expected to show more uniform flow (Fig. 4). The figure shows that, as intended, the manifold with internal baffles guides flow more directly into the channels rather than directing it into the corners of the manifold, as can be seen by the slower (darker blue) pathlines for the original manifold compared to the baffled manifold. Based on the results of the modeling, the original manifold design and the manifold design with internal baffles were recommended for 3D printing. 


\section{Mechanical Modeling and Design}

NASA's micromechanics code MAC/GMC [5] was used to compute the homogenized properties of the electrode and manifold materials accounting for the inherent porosity as well as the laser-etched channels in the anode and cathode layers. The electrolyte with electrodes layer is made of yttria stabilized zirconia (YSZ), and the interconnect layer is made of co-doped lanthanum chromite. These material properties were then used in a finite element analyses of the stack. A finite element model with approximately 49,000 solid elements was created as shown in Fig. 5. ABAQUS general-purpose finite element program [6] was used to perform stress and modal analyses with this model of the stack.

Mechanical analyses were first performed with the stack structure subjected to a uniform operating temperature of $850{ }^{\circ} \mathrm{C}$. This resulted in stresses in various components due to thermal expansion mismatch. These analyses were performed with the original manifold design (i.e. with no internal baffles). The maximum stresses were approximately $80 \mathrm{MPa}$ at a location where the bottom interconnect layer rests on the rectangular manifold.

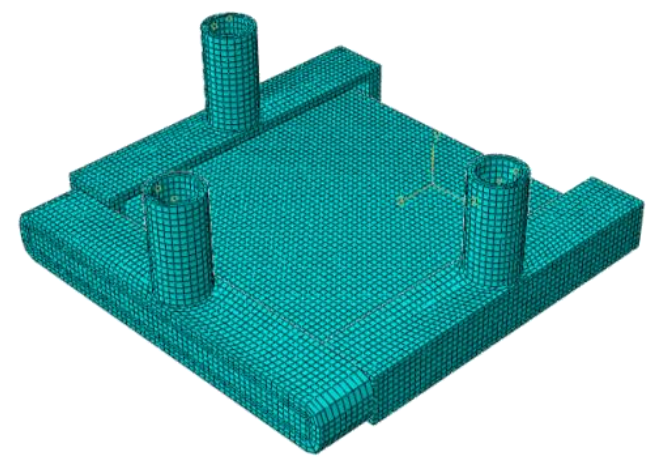

Fig. 5 Finite element model of the 3-cell stack for mechanical analysis.

Modal analyses were also performed on the stack structure. The first natural frequency is on the order of $1800 \mathrm{~Hz}$. This is a relatively high value, but not unexpected considering the very low mass and very high stiffness of the stack. To ensure that the stack can survive the launch loads, a $6.8 \mathrm{~g}$ load (standard practice for space flight structures) was applied in all three directions, one at a time, and a quasi-static analysis was performed for each direction. It was assumed that no external mass is attached to the stack nor external load applied. The highest stresses occurred where the manifold tubing attaches to the main body. However, these stresses are very minimal $(<5 \mathrm{MPa})$. The location of these stresses is shown in Fig. 6. Based on the results of these analyses, stresses appear to be within the allowable stress range of these materials.

\section{Manifold Fabrication and Testing}

In order to remove restrictions on the internal geometry during the design, additive manufacturing was targeted for manifold fabrication. AM of ceramic parts lags behind that of metals, and experience with zirconium oxide is behind that of other softer, lower temperature ceramics such as aluminum oxide. Unlike metals and plastics where lasers are used during additive manufacturing to soften or locally melt material, the high melting temperature of zirconium oxide (approximately $2700{ }^{\circ} \mathrm{C}$ ) requires a different approach. Additionally, use of lasers to achieve high temperatures causes microcracking due to the material's very low thermal conductivity. AM of ceramics typically involves synthesis of binder-coated powders or the separate addition of binder, the formation of an unfired, or 'green' body, and its subsequent drying and firing at high temperatures to remove the binder and sinter the ceramic, resulting in a hard, densified part. Sintering results in up to 20 percent shrinkage, introducing challenges of material distortion and cracking if not properly controlled. Flaws introduced at any step in the process can damage the final ceramic body. Constant coordination between designer and fabricator is necessary to ensure a functional design that also lends itself to ease of additive manufacturing.

After an extensive search of additive manufacturing companies and institutes, a company was found with some experience in manufacturing with zirconium oxide. The original manifold design and the baffled design were sent to the company for fabrication. After reviewing our design, the manufacturer recommended trimming off the unused 


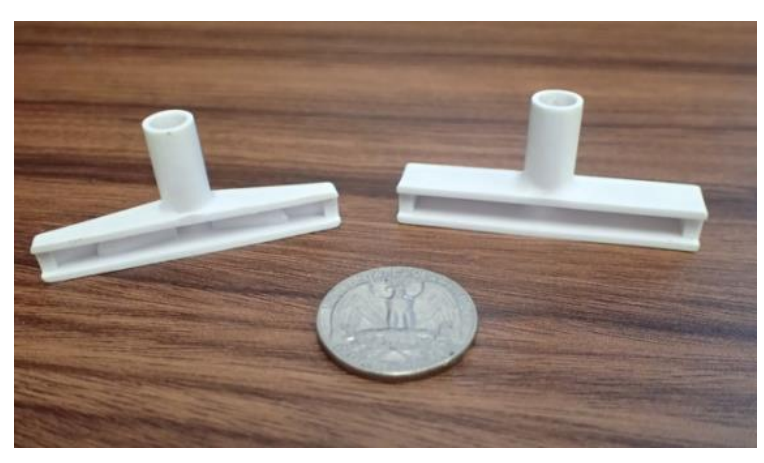

Fig. 7 3D printed zirconium oxide manifolds. New baffled design (left) and original design (right). back corners of the baffled design in order to reduce solid mass and improve manufacturability. Three pieces of the original manifold (sufficient for one stack) and six pieces of the baffled design (for two stacks) were produced. Figure 7 shows two 3D-printed manifolds, one of the original design and one with a baffled design. Some problems with the baffled manifold, including demolding of the weak green parts and achieving the required exacting dimensions after the final firing, presented challenges that were eventually solved by the manufacturer. After the initial nine manifolds were delivered, the NASA team discussed further design changes with the manufacturer that would not affect the internal flow passages but would further improve the manufacturability. After completing the agreed-upon design modifications, an additional set of 3 baffled manifolds were fabricated and delivered to NASA.

To test for any differences in flow uniformity with the two manifold designs, three stacks with attached manifolds were assembled. One original manifold was attached to one 3-cell stack, and two baffled manifolds were attached to two 3-cell stacks (to test for repeatability). All flow tests were performed at ambient conditions. Carbon dioxide was flowed at 200 to 600 standard cubic centimeters per minute $(\mathrm{sccm})$ through each stack and the velocity profile at the exit plane was measured using a hot-wire anemometer. A translating table was used to move the anemometer probe across the exit plane in $2 \mathrm{~mm}$ increments. Because of the fluctuations in the exit plane flow field, the time constant on the probe was set at 20 seconds to provide an average relative velocity value, and the probe was held at each location for several minutes to ensure the reading was at steady-state. Figure 8 provides two views of a baffled-manifold stack and the anemometer set-up in the lab.
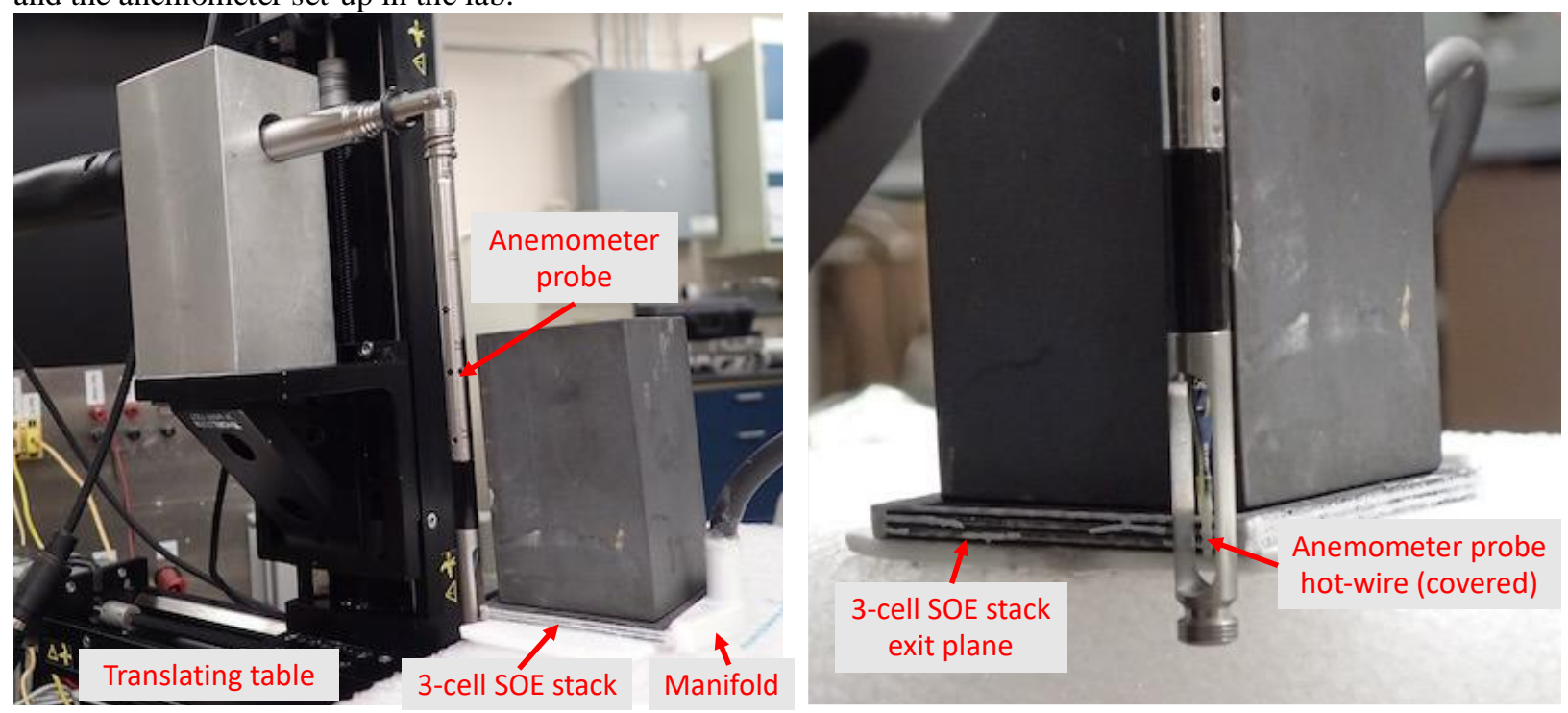

\section{Fig. 8 Two views of the 3-cell stack and anemometer probe set up in the lab in preparation for flow tests.}

It was anticipated that higher exit velocities at the center of the stack would be an indication of poorly distributed flow in the manifold. Initial tests at the nominal flow rate of $200 \mathrm{sccm}$ indicated that the exit velocity was too low to be accurately recorded with the anemometer probe. While data taken at 400 and $600 \mathrm{sccm}$ still showed significant scatter (Fig. 9), some general trends can be seen. At $400 \mathrm{sccm}$, the velocity profiles for all three manifolds were essentially the same, although some additional scatter in the center for the original manifold hints at some starving of the outer channels through the cells. At $600 \mathrm{sccm}$, the scatter is even more severe, and there is again an indication that the original manifold directed more flow down the center of the cells and was less effective at directing flow to the outer channels. While the flow modeling did not predict any differences in exit flow for the two different manifold designs, the model represents an idealized structure, with all of the channels smooth and uniform, and neglecting the 
porosity of the structure. At the scale of the channels in the bi-supported cells, small variations in size could result in larger differences in the predicted flows.
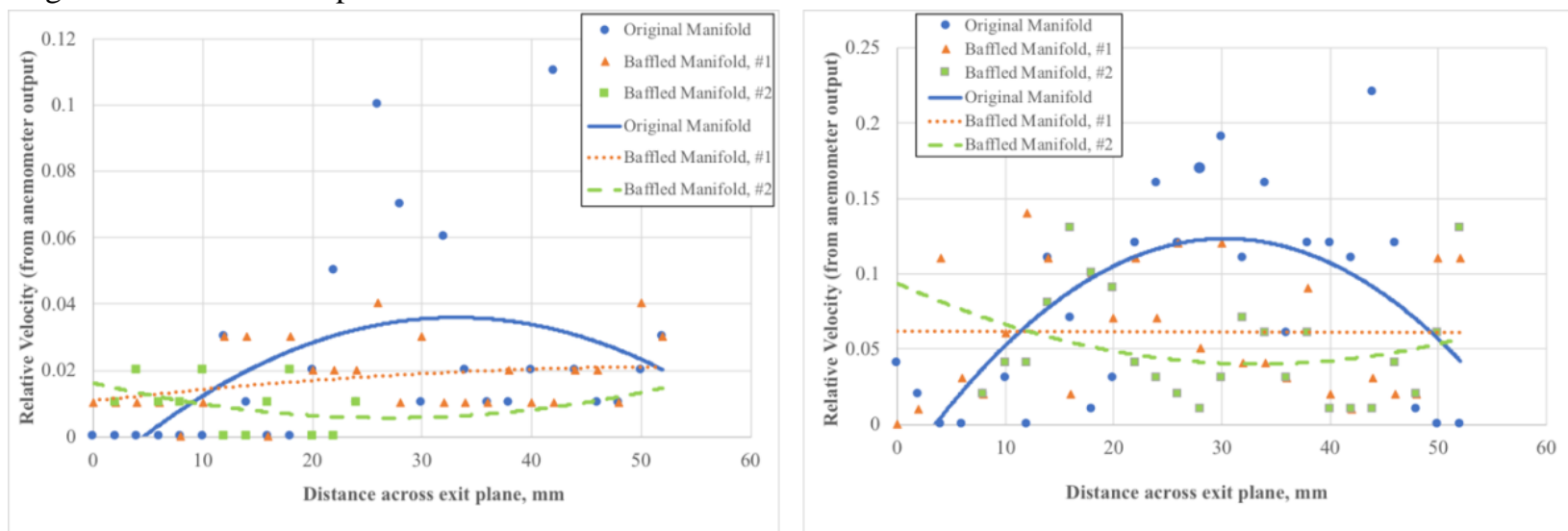

Fig. 9 Relative exit velocity across exit plane for carbon dioxide flow rate of $400 \mathrm{sccm}$ (left) and $600 \mathrm{sccm}$ (right).

\section{Concluding Remarks}

A baffled manifold, produced by additive manufacturing techniques, provided a more uniform flow field across the exit plane of a 3-cell bi-supported solid oxide electrolyzer stack. Large data scatter and slightly higher exit velocities in the center of the exit plane for the stack with a simple, open manifold implies some possible tunneling of the gas flow down the center of the stack. Fluid modeling indicated that the gas flow in the baffled manifold also had a shorter residence time in the manifold compared to the original open manifold design where the inlet gas would linger in the corners.

Mechanical modeling of the 3-cell stack at operating temperature and under simulated launch loads predicted very modest stress values of $80 \mathrm{MPa}$ and less than $5 \mathrm{MPa}$, respectively. These are well within the allowable range for the materials used and provide confidence in the robustness of this all-ceramic design.

The results of the fluid and mechanical modeling and the flow tests indicate that the efficiency of the manifolds for the bi-supported cell design can be improved through the use of additive manufacturing techniques.

\section{References}

[1] Drake, B.G. (editor), "Human Exploration of Mars Design Reference Architecture 5.0,” NASA-SP-2009-566, July 2009.

[2] Drake, B.G., "Human Exploration of Mars Design Reference Architecture 5.0, Executive Summary," February 2009 (available from http://ntrs.nasa.gov/archive/nasa/casi/ntrs.nasa.gov/20090012109.pdf).

[3] Minh, N. Q., "Solid oxide fuel cell technology - features and applications," Solid State Ionics, Vol. 174, No. 1-4, 2004, pp. 271-277.

[4] Green, R. D., Matter, P. H., Beachy, M., Holt, C., Gaydos, J., Farmer, S. C., and Setlock, J. A., "Development Status for a Combined Solid Oxide Co-Electrolyzer and Carbon Formation Reactor System for Oxygen Regeneration", AIAA 2016-5454, Proceedings, AIAA SPACE 2016, Long Beach, CA, 13-16 September 2016.

[5] Bednarcyk, B.A., and Arnold, S.M., "MAC/GMC 4.0 User's Manual, Volume 2: Keywords Manual”, TM 2002-212077/Vol 2, 2002.

[6] Dassault Systems Simulia Corp., 2013: Abaqus Analysis User’s Manual, Version 6.13-1. 